\title{
BMJ Open Understanding influences on the uptake of pulmonary rehabilitation in the East of England: an Inclusive Design/mixed- methods study protocol
}

Yuanyuan Liu, ${ }^{1}$ Terry Dickerson, ${ }^{1}$ Frances Early, ${ }^{2}$ Jonathan Fuld, ${ }^{2}$ P John Clarkson ${ }^{1}$

To cite: Liu Y, Dickerson T, Early $\mathrm{F}$, et al. Understanding influences on the uptake of pulmonary rehabilitation in the East of England: an Inclusive Design/mixed-methods study protocol. BMJ Open 2018;8:e020750. doi:10.1136/ bmjopen-2017-020750

- Prepublication history and additional material for this paper are available online. To view these files, please visit the journal online (http://dx.doi org/10.1136/bmjopen-2017020750).

Received 22 November 2017 Revised 9 March 2018 Accepted 20 March 2018
Check for updates

${ }^{1}$ Department of Engineering, Cambridge Engineering Design Centre, University of Cambridge, Cambridge, UK

${ }^{2}$ Centre for Self Management Support, Cambridge University Hospitals NHS Foundation Trust, Cambridge, UK

Correspondence to

Yuanyuan Liu; yl528@cam.ac.uk

\section{ABSTRACT}

Introduction 1.2 million people in the UK have chronic obstructive pulmonary disease (COPD) that causes breathlessness, difficulty with daily activities, infections and hospitalisation. Pulmonary rehabilitation (PR), a programme of supervised exercise and education, is recommended for patients with COPD. However, only 1 in 10 of those who need it receive PR. Also, the UK National COPD Audit Programme concluded that the COPD treatment might not be accessible to people with disabilities. This paper applies an Inclusive Design approach to community-based PR service provisions. It aims to inform improvements to the PR service by identifying barriers to the uptake of PR in the COPD care journey in relation to patients' capabilities that can affect their access to PR.

Methods and analysis The protocol includes four steps. Step 1 will involve interviews with healthcare professionals and patients to gather insight into their experiences and produce a hierarchical task analysis of the COPD care journeys. Step 2 will estimate the service exclusion: the demand of every task on patients' capabilities will be rated by predefined scales, and the proportion of the population excluded from the service will be estimated by an exclusion calculator. Step 3 will identify the challenges of the PR service; a framework analysis will guide the data analysis of the interviews and care journey. Step 4 will propose recommendations to help patients manage their COPD care informed by the challenges identified in step 3 and refine recommendations through interviews and focus groups.

Ethics and dissemination The Cambridge Central Research Ethics Committee gave the study protocol a positive ethical opinion (17/EE/0136). Study results will be disseminated through peer-reviewed journals, conferences and the British Lung Foundation networks. They will also be fed into a Research for Patient Benefit project on increasing the referral and uptake of PR.

\section{INTRODUCTION}

Underutilisation of pulmonary rehabilitation (PR) Chronic obstructive pulmonary disease (COPD) is a progressive lung disease in which people experience breathlessness, exercise incapacity and vulnerability to
Strengths and limitations of this study

- This protocol uses a novel Inclusive Design method and tools to identify and quantify health service exclusion and make recommendations for improving the accessibility of community-based pulmonary rehabilitation (PR) services.

- Representative healthcare professionals and patients were involved in all aspects of the protocol development to ensure a systematic representation of the real care processes and identification of real issues.

While there are many factors that influence the uptake of PR, this study focuses on patients' individual capabilities in relation to accessing the service.

- Although PR forms the basis of this protocol, the focus could be modified for application to other healthcare services, particularly for community-based treatments of other long-term conditions.

- The Inclusive Design methods and tools consider physical and cognitive exclusion, while other types of exclusion, for instance social or psychological, are not included in the analysis.

exacerbations, frequently requiring hospitalisation. According to the WHO estimates, 65 million people have moderate to severe COPD. ${ }^{1}$ Globally, it is estimated that about 3 million deaths were caused by the disease in 2015 (ie, $5 \%$ of all deaths globally in that year). Approximately 1.2 million people live with $\mathrm{COPD}^{2}$ in the UK and a large proportion of them are older people. This condition costs the UK National Health Service (NHS), a publicly funded national healthcare system, $£ 800$ million per year, mostly relating to hospital admissions. ${ }^{3}$ The UK National Institute for Health and Care Excellence recommends that PR, providing supervised exercise and education, can be offered to patients functionally disabled by COPD. ${ }^{4}$ PR improves symptoms, exercise capacity and quality of life $^{5}$ and leads to fewer repeating exacerbations requiring admission or hospital 
attendance. ${ }^{6}{ }^{7}$ It plays an important role in fostering self-management skills. ${ }^{8}$

The National COPD Audit Programme ${ }^{9}$ estimated that the number of patients with COPD eligible for PR in England and Wales in 2013/2014 was 446 000; however, despite evidence-based guidelines, ${ }^{10}{ }^{11}$ there were only 68000 referrals (15\% of normative need) during that period, and only $69 \%$ of those attended an initial assessment ( $10 \%$ of normative need) ${ }^{9}$ Referral and attendance figures in the East of England (EoE) are not available, but we have no reason to believe that the local figures are significantly different from the national figures. The audit highlighted the need to improve referral and uptake rates. It recommended that PR referral pathways, healthcare professional (HCP) training, information for patients and referrers and barriers to patient access should be reviewed. The audit also demonstrated that the availability of COPD treatment for the full range of severity of disability is not inclusive. ${ }^{12}$

\section{The evidence gap}

Several studies have been carried out to identify barriers that prevent access to PR. ${ }^{13-21}$ Most of these barriers have been identified as relating to the context or environment, people's knowledge and patients' and clinicians' beliefs. ${ }^{22}$ For example, referral to PR can be influenced by a difficult referral process and a lack of knowledge about PR, while uptake can be affected by the lack of transport and geographic distance to a programme, as well as the quality of the HCP's conversation with patients about PR. There is little understanding of how patients' own physical and cognitive capabilities (including mobility, dexterity, reach and stretch, vision, hearing, thinking and communication) relate to their ability to access PR and affect implementation of the care service. People's personal capability to access a healthcare service is a prerequisite for them to use it. Moreover, patients with COPD are likely to have limited mobility. Hence, it is very important to consider patients' capabilities in order to improve access to PR services. Insight into the capability demand within the COPD care pathway (ie, the demands that the care pathway makes on people's capability) would help care providers to better understand the needs of patients and to support their engagement in PR. Therefore, this research will focus on understanding people's capability-related needs while accessing PR within the community.

\section{Study aim}

The aim of the study is to provide recommendations for how primary care PR services in the EoE could improve and increase patients' access to PR services. An Inclusive Design approach will be used to estimate the system demands on patients with COPD and evaluate PR services' exclusion, identifying ways in which the care pathway excludes patients and ways in which the care pathway design can be modified to provide inclusive access to PR for as many eligible patients as possible.
The research question is: how can Inclusive Design be used to improve patients' access to PR services within the community?

\section{METHODS AND ANALYSIS \\ Overview of methods}

Three central concepts will be characterised, namely, (A) Inclusive Design; (B) people's capabilities; and (C) the care pathway/journey. In addition, the link between Inclusive Design and patients' access to PR will be considered.

\section{Inclusive Design}

Inclusive Design can be defined as: (1) 'the design of mainstream products and/or services that are accessible to, and usable by, people with the widest range of abilities within the widest range of situations without the need for special adaptation or design', ${ }^{23}$ and (2) ensuring that the demand made on an individual in a given environment does not exceed their capability to respond. ${ }^{24}$ In this study, Inclusive Design refers to the latter definition.

The Inclusive Design approach is a rigorous user-centred approach, where the fundamental premise is that accessible and usable products or services can only be developed or implemented by first knowing the intended users. ${ }^{25}$ By understanding the user's capability demand in a healthcare context, it is easier to understand their capability-related needs for care and enhance the implementation of the healthcare services.

\section{People's capabilities}

Capabilities in this context refer to people's abilities to access health services. There are mainly two factors that cause a change in people's capability: one is an age-related change in capability, and the other is a change in condition-related capability. Regarding patients with COPD, a large proportion are older people ${ }^{2}$ and the condition of COPD, as well as the ageing process, may significantly impact their capability. People with COPD may be more frail, weaker and have reduced exercise and activity levels. ${ }^{26}$ Moreover, they may be burdened by high levels of anxiety and depression as well as recognised cognitive impairment, particularly at the time of exacerbation. ${ }^{27}$ Many patients have significant differences in their functioning compared with peers. While some of these manifestations relate solely to the presence of COPD, multimorbidity is common and clearly could further impact on patients' capabilities.

\section{Care pathway/journey}

'Care pathway' or 'care journey' are both terms used to describe the process of healthcare service delivery. Care pathways are more generic and described from an organisational perspective. A pathway may take the form of an integrated management plan that provides a sequence and timing of actions necessary to achieve a standard care process and optimal efficiency for clinicians. ${ }^{28}$ 


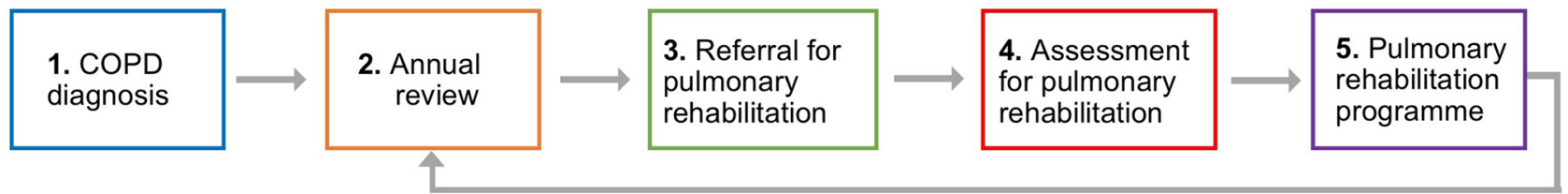

Figure 1 The main stages in the NHS primary care pathway of COPD rehabilitation are: (1) COPD diagnosis, (2) annual review, (3) referral for pulmonary rehabilitation, (4) assessment for pulmonary rehabilitation and (5) pulmonary rehabilitation programme. COPD, chronic obstructive pulmonary disease; NHS, National Health Service.

For example, the main stages in the NHS primary care pathway of COPD rehabilitation are shown in figure 1.

A patient's care journey refers to the process that he or she goes through in order to receive the care. As shown in figure 2 , we define the care journey as a series of tasks. For example, the first stage in figure 1, that is, COPD diagnosis, can be further broken down into four tasks: (1) make an appointment with a general practitioner (GP); (2) go to the GP practice; (3) have a GP consultation; and (4) obtain a diagnosis. The detailed care journeys that patients experience in accessing the PR service would be more easily identified in the context of a deeper understanding of COPD services.

\section{The connection between Inclusive Design and patients' access to PR}

Any service makes demands on patients, and patients have to have sufficient capabilities in order to respond to these demands and access the service (figure 3) ${ }^{29}$ If the demands of accessing the PR service exceed the capabilities of the patients, then exclusion or difficulty in using the service will arise. Taking the task 'go to the GP practice' as an example, if the home of a patient with COPD is relatively far from the GP surgery and the patient's mobility is limited, then he or she may not be able to get to the GP practice. In this case, this patient is excluded from the PR service as he or she cannot even see the doctor. With a better understanding of the tasks in the patient care journey, we are able to estimate the service's

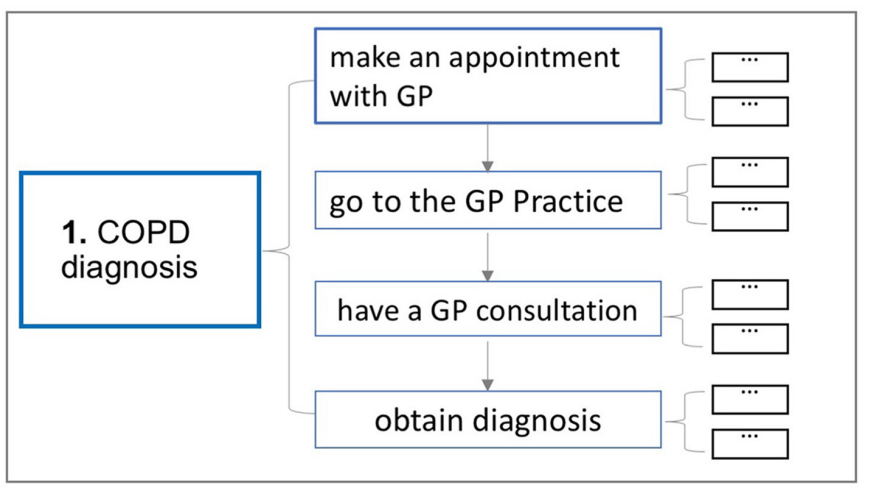

Figure 2 Care journeys consist of specific tasks. The first stage of the NHS primary care pathway of COPD rehabilitation, COPD diagnosis, can be further broken down into four tasks: (1) make an appointment with a general practitioner (GP); (2) go to the GP practice; (3) have a GP consultation; and (4) obtain a diagnosis. COPD, chronic obstructive pulmonary disease. system demands on patients' capabilities and analyse the extent of system inclusion.

\section{Study procedures}

As illustrated in figure 4, the research is divided into four steps: semistructured interviews will be used in step 1 (mapping pathway); step 2 (estimate exclusion) and step 3 (identify challenges) are mainly data analysis, and interviews and focus groups will be used in step 4 (propose recommendation).

\section{Step 1: mapping pathway}

\section{Interviews with HCPS}

We will conduct interviews with HCPs (including GPs, practice nurses and physiotherapists) who refer patients to PR, as well as PR service managers and healthcare commissioners (see section sampling and recruitment for details).

The interviews with HCPs will gather insight into their experiences and perception of the COPD care pathway. The main stage of the NHS primary care pathway (see figure 1) will be presented to HCPs, and the detailed primary care clinicians' pathways for PR will be identified based on the interview data. This understanding of the care pathway from the clinicians' perspective will be used to help map patients' care journeys. Specifically, we will focus on the pathway from diagnosis to PR programme attendance, while recognising the number of possible different routes to PR referral. Patients' ability to do exercises and take part in PR once they have accessed the service is not the focus of this research. The questions are shown in online supplementary appendix 1.

\section{Interviews with patients}

Three categories of patients with COPD will be interviewed: patients who have accepted a PR offer, patients who have declined a PR offer and patients who have never been referred to PR (see section sampling and recruitment for details).

The interviews with patients with COPD will capture the detailed COPD care journey from patients' perspectives, gather insight into patients' experiences of PR and their care needs and assess the capabilities of patients with COPD. The questions are shown in online supplementary appendices 2 and 3 .

Data analysis: (1) transcriptions and field notes will be managed and analysed using NVivo software. (2) The detailed care journeys that patients go through to receive COPD treatment and the detailed care pathway that HCPs engage in when delivering COPD treatment 


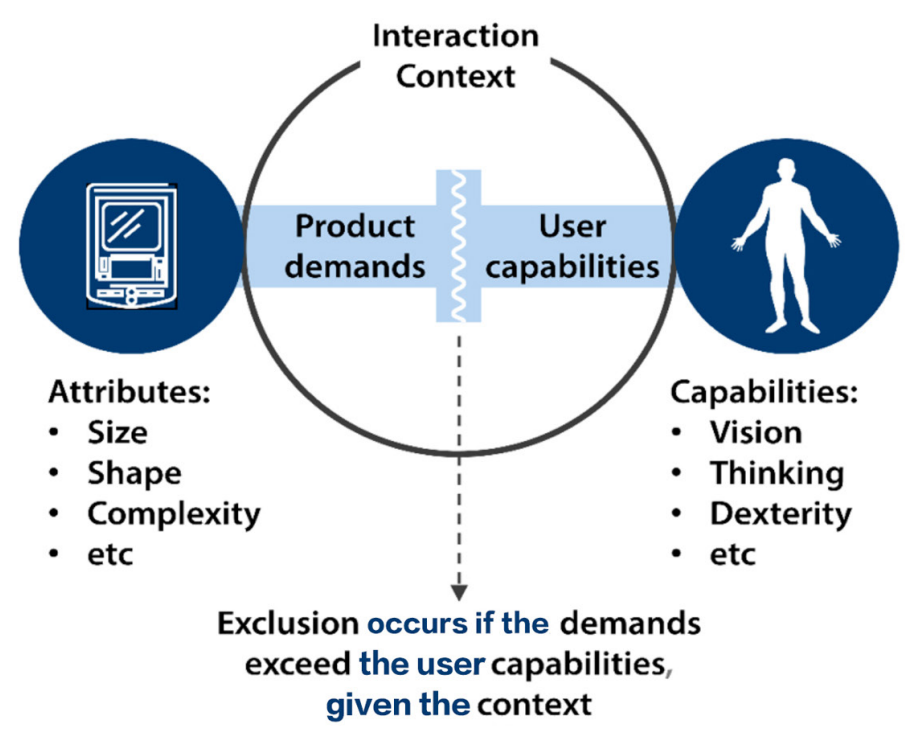

Figure 3 Interaction context-Demand and Capabilities (Persad et $a{ }^{29}$ ). The example is of a physical product, but this applies equally to services. Reproduced from www.inclusivedesigntoolkit.com with permission.

will be summarised and represented as a hierarchical task analysis $^{30}$ (figure 5). For example, the stage 1 COPD diagnosis in figure 1 could be further broken down into the following steps: make an appointment with a GP, go to the GP Practice, have a GP consultation and obtain diagnosis (figure 2). Making an appointment with a GP can be done by telephone or computer, could be booked by dropping in or could be booked by others. Making an appointment by telephone can be further broken down into the following steps: make a telephone call, request an appointment, agree on details and mark in diary. (3) The similarities and differences between the care pathway from the HCPs' perspectives and the care journey from the patients' perspectives will also be explored.
Output: a map of the patients' care journey will be defined. This will be used in step 2 to estimate the reasons and levels of exclusion.

\section{Step 2: estimate exclusion}

Estimate how demanding the COPD care journey is for patients

Tasks will be analysed to determine the demands of the COPD care journey on patients. Specifically, the demand of every task will be rated by predefined scales. These scales were constructed based on the questions in the Disability Follow-up to the Family Resources Survey, which was originally performed to help plan welfare support for disabled people. ${ }^{31}$ The survey of more than 7000 people included many with respiratory conditions

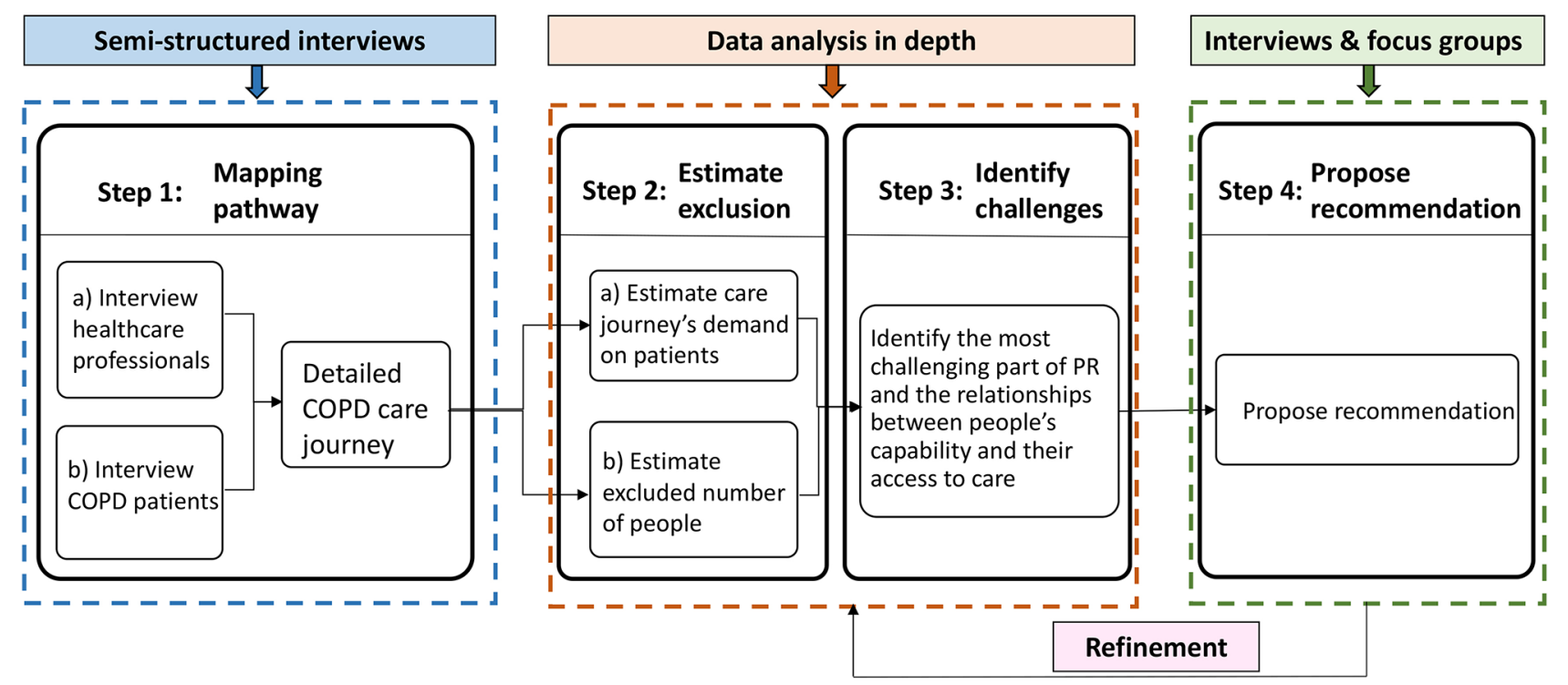

Figure 4 Study design. COPD, chronic obstructive pulmonary disease. 


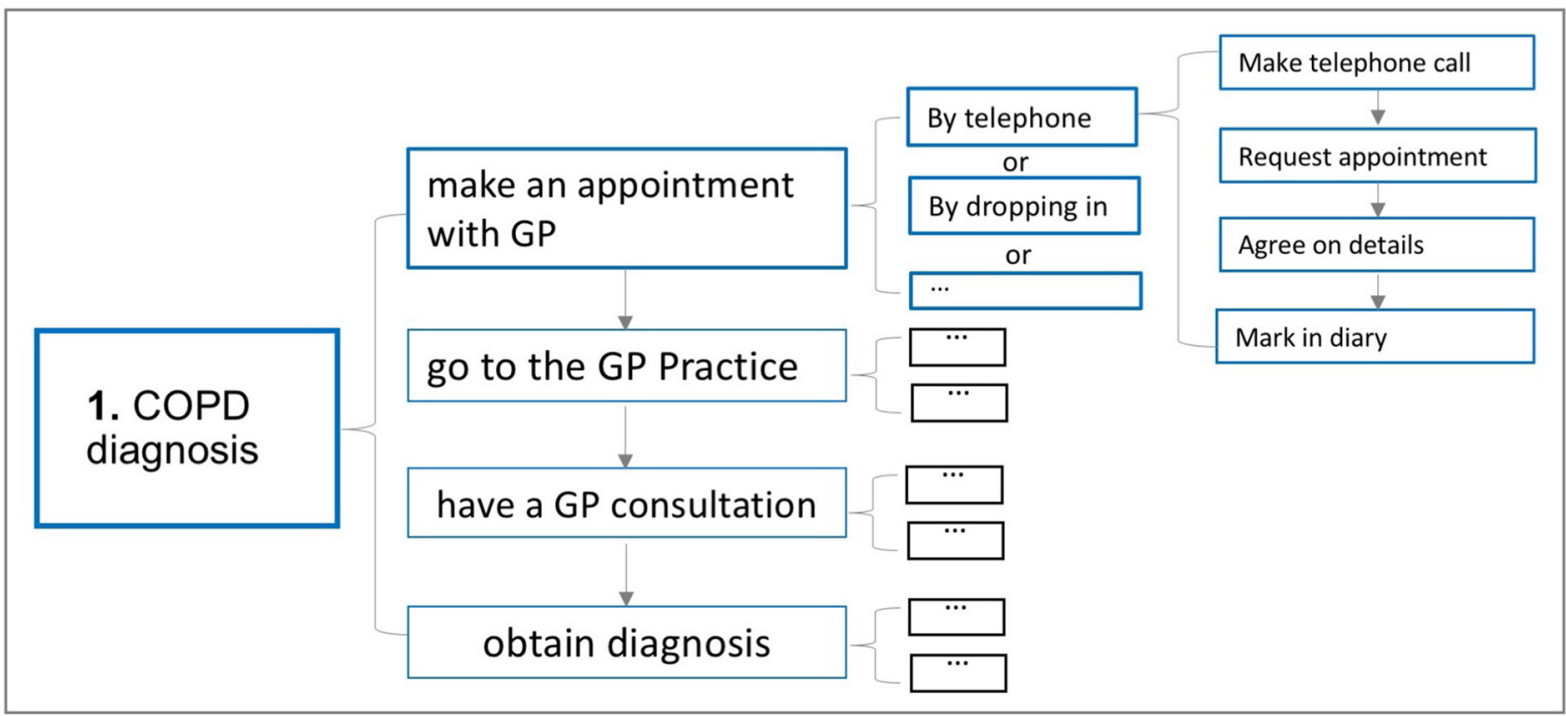

Figure 5 Example for care journey represented in a hierarchical task analysis, not all tasks and sub tasks are shown for clarity. COPD, chronic obstructive pulmonary disease; GP, general practitioner.

including COPD. Subsequently, these data have been used to assess numerous products and services. ${ }^{31-33}$ Examples of assessing hearing, reach and speech comprehension demand scales are shown in figure 6 . Higher demand on peoples' capabilities causes higher scale ratings, and the symbol '>' off scale means it is excessive for a mainstream service.

With the predefined scales, the demand on every task will be assessed. For example, the task 'to make a telephone call' places demands on patients' sensory capabilities (to hear clearly throughout the call and see the number buttons), motor capabilities (to hold the phone and press the number buttons) and cognitive capabilities (to remember/know the telephone number to call and communicate with the receptionist). The demand of this task on patients' hearing capability is close to the scale 8 , that is, use telephone without special adaptations for hearing impairment, so the hearing demand is rated scale 8 (figure 7 ). The level of the tasks' description is matched against the details of predefined scales.

\section{Estimate the number of people excluded from PR on the basis of their capabilities}

The 'Exclusion Calculator' (an Inclusive Design tool) produced by the University of Cambridge Engineering Design Centre uses a large database of British users with a range of disabilities to estimate the proportion of the British population (Great Britain) that is unable to use a product or service because of the demands that it places on the users' capability. ${ }^{34}$ A version of this calculator is freely available on the Inclusive Design Toolkit website. ${ }^{34}$ By inputting the estimated demands of each task along the COPD care journey, the 'Exclusion Calculator' can estimate the number of people within general population excluded from accessing PR (in every task and on the whole care journey). Figure 8 shows an
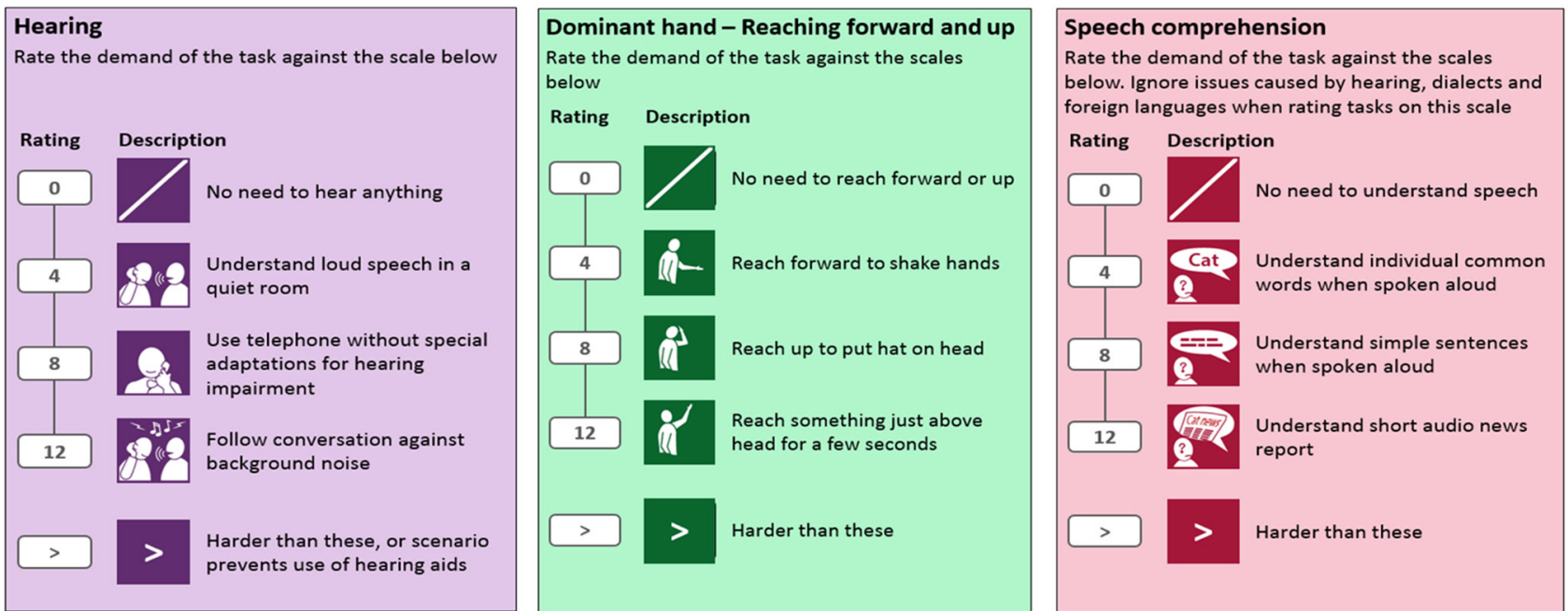

Figure 6 The standard of measuring hearing (left), reaching forward and up (middle) and speech comprehension (right). Examples of assessing hearing, reach and speech comprehension demand scales are shown. 


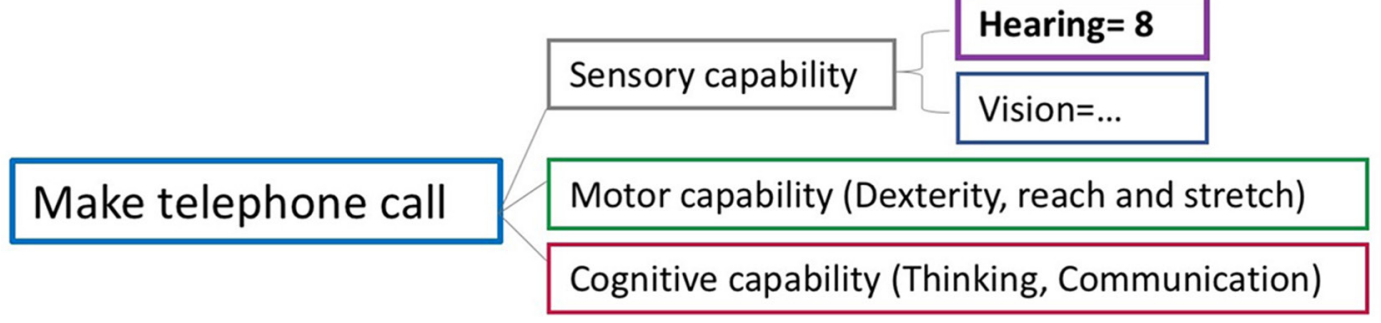

Figure 7 Demand on capability of making a phone call.

example of measuring the hearing exclusion for the task 'make a telephone call'.

The original population data (sample size $n=7618$ ) is from the Disability Follow-up to the Family Resources Survey. ${ }^{31}$ Among these participants, there were nearly 1000 participants who self-reported that they have a respiratory issue, and it is likely that a significant number of these people could benefit from PR. Although the exclusion numbers from the calculator are based on the general population (rather than those with COPD), we can still obtain insights about challenges raised by the COPD care journeys from the exclusion levels predicted. For some tasks, the calculator will overestimate the level of exclusion but still give useful insights as a loss in capability may not be due to COPD. Nonetheless, changes in the demand of PR on the patients with COPD will be reflected by changes in exploration from the whole population.

Output: the patient care journey map, enhanced by the types and levels of exclusion, will be used to evidence the relationship between people's capabilities and their ability to access PR in step 3.

\section{Step 3: identify challenges}

Understand the most challenging part of PR and analyse the relationship between people's capabilities and their access to PR services. The interviews, rated demand and the excluded number of people will provide clues to identify the most challenging issues linked to PR and develop initial recommendations. It will also help to analyse the relationships between people's capabilities and their potential to access PR.

Data analysis: an inductive Framework Analysis ${ }^{35}$ will be used to structure the data analysis (including interview transcript and exclusion data). First, initial categories/themes will be identified and put in the coding matrix; then, the relationship among the different coded data will be analysed. Finally, the core categories to propose the initial recommendations will be established.

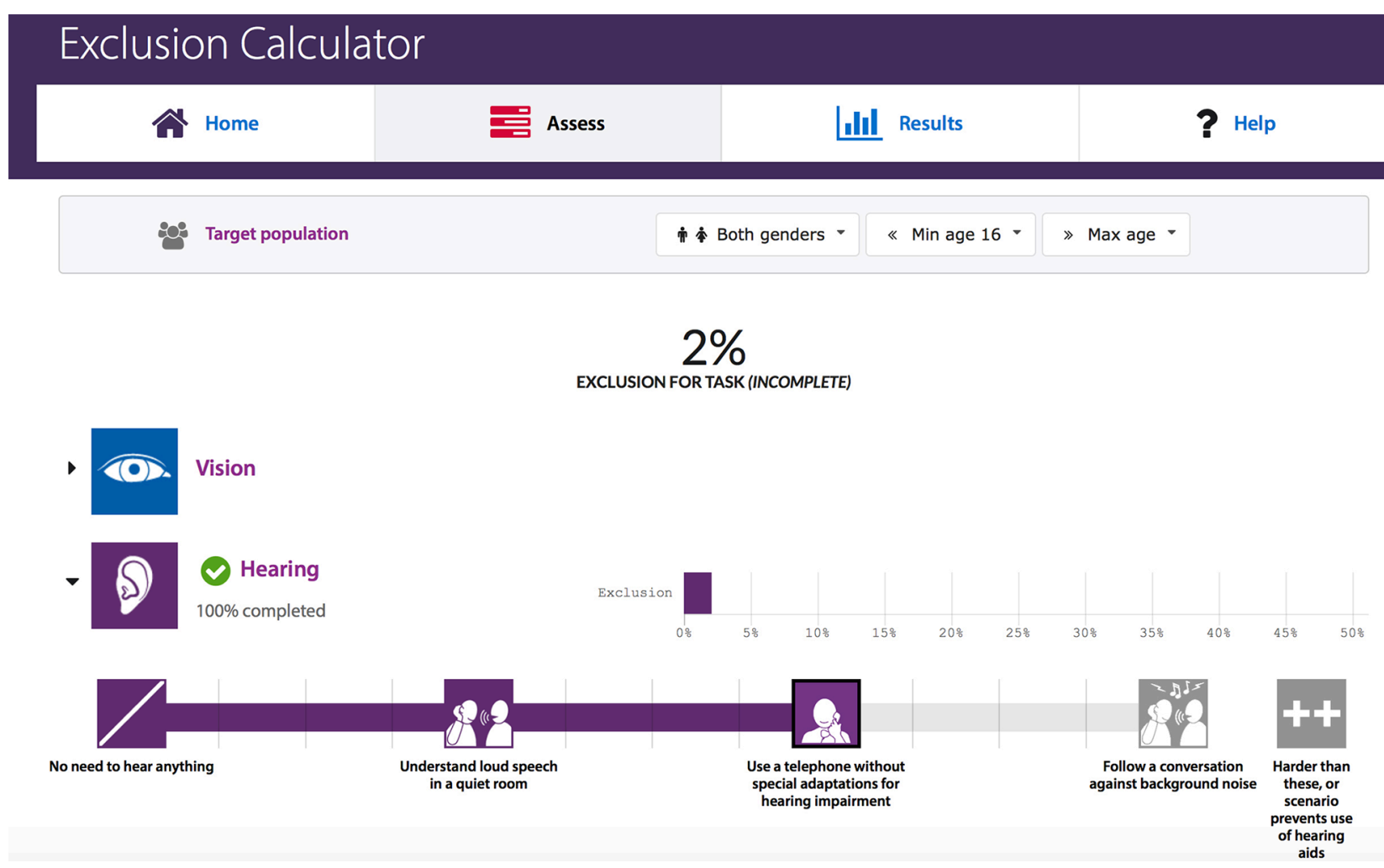

Figure 8 Calculating exclusion (ie, the hearing exclusion for the task 'make a telephone call'). 
Output: the initial recommendations that helps identify patients' needs will be proposed, which contains a set of questions that prompts patients to consider their potential needs along their COPD care journey, in particular those needs that are caused by reduced capabilities when trying to access PR. The initial recommendations will be refined in step 4 .

\section{STEP 4: propose recommendation(s)}

Propose and refine recommendations that aims to help patients understand their needs and manage their COPD care. These recommendations could be used as an interactive tool between HCPs and patients to help HCPs to better understand patients' needs, especially the capability demand within the process of PR. (1) Further interviews with HCPs (including GPs, practice nurses or physiotherapists) will be conducted to obtain the views of HCPs on the proposed recommendations. Potential participants (new or existing interviewees) will be invited to participate by email or via a phone call. (2) Two focus groups will be organised that include both patients with COPD and HCPs to obtain the views of patients and HCPs on the proposed recommendations. (3) The recommendations will be further refined based on the data from the interviews and focus groups.

Data analysis: the interview and focus group data will be transcribed and coded in an inductive coding structure to help refine the recommendations.

Output: the data analysis from the interviews and focus groups will help to better understand the needs of patients with COPD accessing PR. The refined recommendations that contains the key points of the COPD care journey will be available to inform the delivery of the PR service.

\section{Study setting}

Interviews with HCPs will take place at their place of work or other NHS premises by arrangement.

Interviews with patients will take place on community premises where PR classes are conducted, where Breathe Easy group meetings are held or at a location of choice (ie, the Engineering Department of University of Cambridge or their home with someone accompanying the researcher or in a coffee shop). Interviews that are conducted in a PR setting will be on a one-to-one basis to minimise any bias or any external influence. Overall, the researchers will need to be pragmatic about the timing, location and other environmental factors; however, the analysts will formally reflect on the influence of contextual factors on the interpretation of the results.

\section{Sampling and recruitment \\ Sampling sites}

Sampling will be carried out in a single region, the EoE in the UK, that covers both urban and rural areas. The sites available to the researchers will be limited; however, sites in both urban and rural setting will be included to encourage a broad selection of professionals and patients to enrol in the study.

\section{Eligibility criteria}

- HCPs who can refer to PR programmes from their primary care annual review, physiotherapists who provide $P R$, PR service managers and the CCG strategy managers in Cambridgeshire and Peterborough.

- People resident in the EoE, aged 18 years or over, with a diagnosis of COPD, free from exacerbation or hospitalisation within the preceding 4 weeks, eligible for PR as defined by the guideline recommendations and able to read/write in English.

\section{Size of sample}

Warren ${ }^{36}$ suggested that the minimum number of interviews needs to be between twenty and thirty for an interview-based qualitative study to be published. ${ }^{37}$ Mason reported that the interview number range was 1-95 (with a mean of 31 and a median of 28) for interview-based qualitative studies in doctoral theses. ${ }^{38}$ Adler also advised the best number of people for a qualitative research is between 12 and 60 , with 30 being the mean. ${ }^{39}$ The size of sample in this study will be consistent with these recommendations, and the details are shown in table 1 . The size of the subgroups will be adjusted as necessary to reach data saturation to ensure the validity of the results. This approach complements the sampling techniques as described below.

Table 1 The size of sample

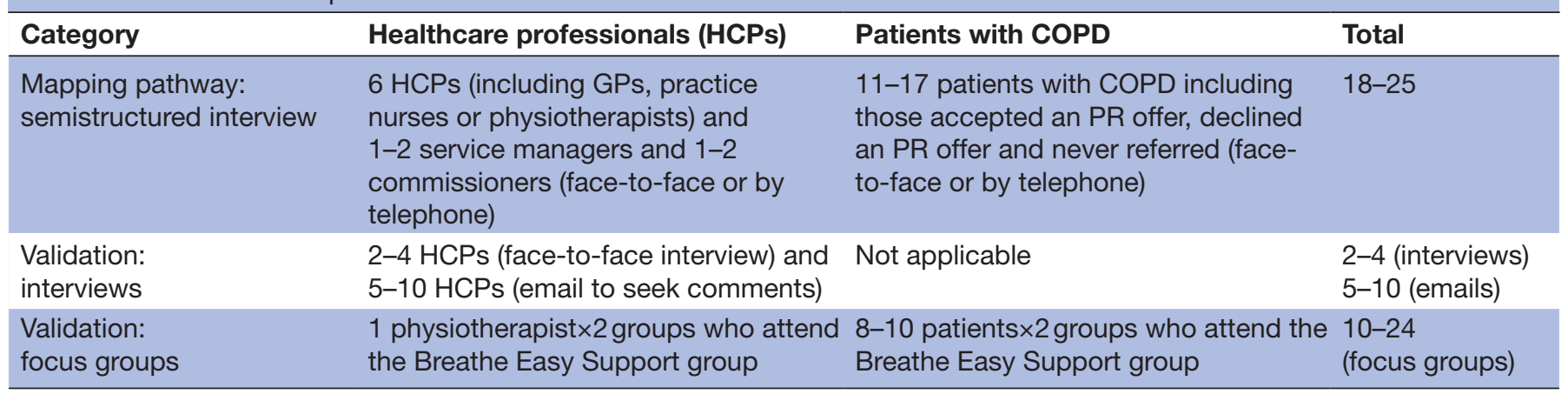

COPD, chronic obstructive pulmonary disease; PR, pulmonary rehabilitation. 
Table 2 Recruitment plan for healthcare professionals

\begin{tabular}{ll}
\hline $\begin{array}{l}\text { Healthcare } \\
\text { professionals' role }\end{array}$ & Understanding of PR pathway \\
\hline $\begin{array}{l}\text { GPs/practice nurses } \\
(n=6)\end{array}$ & $\begin{array}{l}\text { Familiar with the process of diagnosis, } \\
\text { annual review and referral }\end{array}$ \\
$\begin{array}{l}\text { Physiotherapists } \\
(n=4-6)\end{array}$ & $\begin{array}{l}\text { Familiar with the process of referral, } \\
\text { assessment for PR, PR programme } \\
\text { and annual review }\end{array}$ \\
$\begin{array}{ll}\text { PR service manager } \\
(n=1-2)\end{array}$ & $\begin{array}{l}\text { Familiar with the whole PR pathway } \\
\text { process and having connections with } \\
\text { other professionals }\end{array}$ \\
\hline $\begin{array}{l}\text { Commissioners and } \\
\text { manager }(n=1-2)\end{array}$ & $\begin{array}{l}\text { Familiar with the process of designing } \\
\text { or supporting the COPD care pathway }\end{array}$ \\
\hline
\end{tabular}

COPD, chronic obstructive pulmonary disease; PR, pulmonary rehabilitation.

\section{Sampling technique}

Data saturation refers to a process of data collection whereby no substantial new insight data are generated. This sampling strategy will be used to gather data in Cambridgeshire and Peterborough. Sampling will be informed by the interview results to represent a range of care pathways/journeys and their experiences along the care pathways/journeys. A stakeholder map will be used to help identify a representative sample and gather adequate data. The researcher will seek advice from the interviewees to ensure all the key stakeholders are covered. If any role within the stakeholder map is missing or affects the data analysis, the researcher will try to find appropriate interviewees. The detailed plan is shown in table 2 and table 3 (where $n$ refers to the number of people):

\section{Sampling identification}

Access methods have been discussed with Clinical Research Network (CRN) Eastern.

HCPs will be identified by:

i. Working with CRN Eastern locality managers to access HCPs.

ii. Accessing HCPs through visiting or emailing GP practices.

iii. Accessing PR providers through EoE PR network.

In terms of identifying patients with COPD:

i. People who have accepted a PR offer will be identified through PR provider registers.

ii. People who declined a PR offer may be challenging to engage. HCPs and PR providers will be asked to identify people they know, and we will work with the British Lung Foundation to engage this group, for example, through telephone interviews/home visits, or interviews while people are visiting their practice for an annual review.

iii. People who have never been referred to PR will be identified through COPD registers in participating
GP practices. We will sample one GP practice in a city centre and another in a rural area.

\section{Patient and Public Involvement (PPI)}

The aim of our PPI activities is to ensure that we bring patients, accompanying carers and HCPs together to deliver the research collaboratively so that it comprehensively addresses patient needs. Patients' enthusiasm has been encouraged, and we welcome their continuing contribution.

Five patients with COPD have contributed to the research questions, aim, participant sheets, interview schedule, consent forms and the plain English summary. Several discussions were held with three physiotherapists who provided clinicians' perspectives on the research questions, aim, participant sheets, interview schedule and consent forms for HCPs. The lay summary and interview materials (participant information sheets) were reviewed by nine members of the PPI panel, Cambridge University Hospitals. They have confirmed the language used is understandable and have provided useful comments to refine the research.

The study results will be disseminated to study participants by post or email based on their preferences.

\section{Research bias}

The interviews and focus groups will be used to gain multiple perspectives on the understanding of HCPs' and patients' experiences. The research has been carefully designed and has been reviewed by experts to ensure it will inform us of the current challenges that patients experience when accessing PR. The research protocol has been reviewed by the Research Advisory Committee of Cambridge University Hospitals, and advice has been received from the members of the PPI panel, Cambridge University Hospital (NHS Foundation Trust).

To reduce the risk of bias during data analysis, the work will be supervised by a senior researcher. A second researcher will independently analyse the data at each stage of the analysis. The primary and secondary analysts will compare results and resolve any discrepancies. Should any discrepancies not be resolved, the supervising senior researcher shall adjudicate.

\begin{tabular}{|c|c|c|c|}
\hline & $\begin{array}{l}\text { PR } \\
\text { programme }\end{array}$ & $\begin{array}{l}\text { Age } \\
\text { group (years) }\end{array}$ & Capability \\
\hline $\begin{array}{l}\text { People with } \\
\text { COPD }\end{array}$ & $\begin{array}{l}\text { Accepted PR } \\
\text { offer }(n=5-7) \\
\text { Declined PR } \\
\text { offer }(n=3-5) \\
\text { Never referred } \\
(n=3-5)\end{array}$ & $\begin{array}{l}16-49 \\
50-64 \\
65-74(n \geq 5) \\
75+(n \geq 2)\end{array}$ & $\begin{array}{l}\text { Hearing, vision, } \\
\text { mobility, dexterity, } \\
\text { reach and stretch, } \\
\text { communication, } \\
\text { thinking. } \\
\text { Ensure a full range } \\
\text { of capability loss } \\
\text { is covered by the } \\
\text { samples }\end{array}$ \\
\hline
\end{tabular}

PR, pulmonary rehabilitation. 


\section{DISCUSSION}

In this protocol, we describe a novel approach to service improvement that takes a structured and rigorous stance towards analysing barriers to PR referral and uptake. An Inclusive Design approach has been widely used to understand customer diversity and respond to this diversity with informed design decisions. ${ }^{40}$ The Inclusive Design methods used in this study have been adapted from their original context (measuring the exclusion of consumer products) and applied to care journey analysis. Specifically, one of the Inclusive Design tools, Exclusion Calculator, estimates the proportion of the British population who would be unable to use a product or service because of the demands that it places on the users' capabilities. The tool has been widely used in measuring the exclusion of consumer products. For healthcare setting, similar to products setting, it is also important to understand healthcare services' demands on users' capabilities, so that healthcare services can be better developed and benefit more people, that is, more inclusive. A strength of the approach is that it has both qualitative and quantitative perspectives that give insight into how and where changes can be made and what impact the changes might have on the uptake of PR. As the Exclusion Calculator data is sampled based on general British population rather than people with COPD, the exclusion levels may be overestimated, but it can still give clues and insights about the needs of people with COPD. How using general population data affects the results will be subtle, depending on the specific tasks where the greatest exclusion occurs and the capabilities needed to perform those tasks. The analysis will need to apply some care in interpreting the details of the exclusion results.

We focus on the primary care journey for people with COPD. We recognise that referrals for PR stem from a multiplicity of sources (eg, at exacerbation, by community specialist teams, through secondary care). However, the learning applied to one specific route, for example, from an annual review, is likely to identify at least some of the capability issues arising in other routes. This protocol is part of a first study into the application of Inclusive Design to healthcare processes. The authors have published one research that explores the role of Inclusive Design in improving people's access to back pain treatment. ${ }^{41}$ The protocol can be adapted and applied to other PR pathways and indeed pathways for other conditions.

In this study, we enquire about people's self-reported capabilities, rather than test them in practice to determine capabilities. This methodology of seeking self-reported capabilities is validated ${ }^{42}$ and more feasible than extensive field testing.

The protocol focuses on physical and cognitive factors that contribute to preventing people from accessing PR services. We recognise that factors such as mental health, social exclusion and other factors may also affect the uptake of PR. Hence, this protocol is only a partial answer to identifying PR service exclusions. However, as previously stated, it is a prerequisite for accessing a healthcare service in which the service demands made do not exceed the capability of patients.

\section{ETHICS AND DISSEMINATION}

\section{Assessment and management of risk}

Participants may be vulnerable due to age/frailty. In order to manage this risk, they will be identified via GP practices or PR services to whom they are known and who can ensure participants are aware of their diagnosis of COPD prior to the researcher approaching them. Sources of support will be identified to whom participants can be directed.

If there is a concern about a person's health or safety, the researcher may contact the relevant GP; however, as this would mean breaking confidentiality, it would only be done if the person were clearly at risk. When making appointments for patients to attend research activities, researchers will ensure that the time and location are acceptable to the participant.

It is recognised that interviews may involve discussion of sensitive topics regarding the patients' health, lifestyle or quality of life. To address this:

- All study materials, including interview questions, have been developed in collaboration with a PPI group to ensure topics are addressed in a sensitive way.

- Participants will be directed to sources of support and information.

\section{Data protection and patient confidentiality}

The Patient Information Sheet will detail the data to be collected and how it will be stored.

The study will comply with the Data Protection Act, which requires data to be anonymised as soon as it is practical to do so. The study staff will ensure that participants' anonymity is maintained. Staff seeking consent will ask patients only for personal data that confirms their eligibility for the study and at no point will study staff have access to patients' medical records.

On entering the study, participants will be assigned an ID. Participant names will only be identifiable on a cross-referenced list of IDs and names, which will be stored in a locked filing cabinet on Trust premises. When recordings of interviews or focus groups are transcribed, only the ID will be used as an identifier.

Data collected will be entered onto secure computers in the Engineering Design Centre (EDC) at the University of Cambridge and will only be accessible to the study team. Paper documents will be stored in a locked cupboard in the EDC and will only be accessible to the study team.

All electronic files will be password protected and access restricted to the study team. Participants will be identified only by a participant ID number on any electronic database. The name and any other identifying details will not be included in any study data electronic file. Data sharing and storage will meet the requirements of the National Institutes of Health Research. Data will be securely stored in the University of Cambridge Research Repository. 


\section{Ethical approval}

This research has been ethically reviewed and approved by Cambridge Central Research Ethics Committee. The study's REC reference number is $17 / \mathrm{EE} / 0136$. It has two work packages: work package 1 , which uses an Inclusive Design approach is presented above, and work package 2 is to develop a toolkit to increase referral to and uptake of PR in primary care.

\section{Dissemination}

We will work closely with the British Lung Foundation, who have a track record of disseminating innovation through patient networks, publications, online information, service development and HCP engagement. Two academic papers will be published in peer-reviewed journals compliant with policy on open access on: (1) capabilities of patients with COPD and (2) comparison of COPD care pathways between HCPs and patients with COPD. Presentations will be given at regional, national and international academic and professional conferences, for example East Anglian Thoracic Society, British Thoracic Society and European Respiratory Society. The output will also comprise a part of a doctoral thesis by the lead author. The study started in June 2017 and will continue until April 2018.

Acknowledgements The authors would like to acknowledge Lucy Bennett at Cambridge University Hospitals NHS Foundation Trust for her help and support in developing the research; the authors would like to thank the Patient and Public Involvement (PPI) panel of Cambridge University Hospital, patients with COPD and physiotherapists involved in this study, Nicola Cavaleri and Denise Chappell from the Language Unit who helped review and refine the protocol. The Exclusion Calculator was produced by the Cambridge Engineering Design Centre, as part of the ID-3 Inclusive Design Consortium run by the Centre for Business Innovation, which will be used in this research.

Contributors YL initiated the protocol. TLD and PJC provided supervision for the research and reviewed as well as finalised the protocol. FE drafted the background, ethics and dissemination and reviewed the protocol. JF was involved in the design of the protocol, clinical guidance for delivery of the study and revision of the manuscript.

Funding The research is part funded by the NIHR Collaboration for Leadership in Applied Health Research \& Care (CLAHRC) East of England and Buchanan Fund of Downing College. The China Scholarship Council provided funding for the researcher's PhD study.

Disclaimer The views expressed are those of the authors and not necessarily those of the NHS, NIHR or Department of Health and Social Care.

Competing interests None declared.

Patient consent Not required.

Provenance and peer review Not commissioned; peer reviewed by the Research Advisory Committee (RAC) of Cambridge University Hospitals.

Data sharing statement The Inclusive Design methods and tools are available from our website: http://www.inclusivedesigntoolkit.com

Open Access This is an Open Access article distributed in accordance with the Creative Commons Attribution Non Commercial (CC BY-NC 4.0) license, which permits others to distribute, remix, adapt, build upon this work non-commercially, and license their derivative works on different terms, provided the original work is properly cited and the use is non-commercial. See: http://creativecommons.org/ licenses/by-nc/4.0/

(C) Article author(s) (or their employer(s) unless otherwise stated in the text of the article) 2018. All rights reserved. No commercial use is permitted unless otherwise expressly granted.
REFERENCES

1. World Health Organization. Chronic obstructive pulmonary disease (COPD). http://www.who.int/mediacentre/factsheets/fs315/en/ (accessed 20 May 2016).

2. British Lung Foundation. Chronic Obstructive Pulmonary Disease (COPD) statistics. http://statistics.blf.org.uk/copd (accessed 20 May 2016).

3. National Institute for Health and Clinical Excellence. COPD Costing Report. London: National Institute for Health and Clinical Excellence, 2011.

4. National Institute for Health and Clinical Excellence. COPD Guideline CG101. 2010 http://guidance.nice.org.uk/CG101 (accessed 20 May 2016).

5. McCarthy B, Casey D, Devane D, et al. Pulmonary rehabilitation for chronic obstructive pulmonary disease. Cochrane Database Syst Rev 2015;3:1-54.

6. Seymour JM, Moore L, Jolley CJ, et al. Outpatient pulmonary rehabilitation following acute exacerbations of COPD. Thorax 2010;65:423-8.

7. Griffiths TL, Burr ML, Campbell IA, et al. Results at 1 year of outpatient multidisciplinary pulmonary rehabilitation: a randomised controlled trial. Lancet 2000;355:362-8.

8. Singh SJ, ZuWallack RL, Garvey C, et al. Learn from the past and create the future: the 2013 ATS/ERS statement on pulmonary rehabilitation. Eur Respir J 2013;42:1169-74.

9. Royal College of Physicians. National COPD Audit 2013-2016. https ://www.brit-thoracic.org.uk/audit-andqualityimprovement/nat ionalcopd-audit-2013-2016/ (accessed 17 Mar 2016).

10. Bolton CE, Bevan-Smith EF, Blakey JD, et al. British Thoracic Society Pulmonary Rehabilitation Guideline Development GroupBritish Thoracic Society Standards of Care Committee. British Thoracic Society guideline on pulmonary rehabilitation in adults. Thorax 2013;68(Suppl 2):ii1-30.

11. NHS Service Specification. Pulmonary Rehabilitation Service. 2012 https://www.gov.uk/government/publications/commissioning-toolkitfor-respiratory-services (accessed 15 March 2015).

12. National COPD Audit Programme. National organisational audit report-Pulmonary Rehabilitation: Time to breathe better. 2015 https:// www.brit-thoracic.org.uk/standards-of-care/audit/national-copdaudit-programme/ (accessed 20 May 2016).

13. Harris D, Hayter M, Allender S. Factors affecting the offer of pulmonary rehabilitation to patients with chronic obstructive pulmonary disease by primary care professionals: a qualitative study. Prim Health Care Res Dev 2008;9:280-90.

14. Johnston K, Grimmer-Somers K, Young M, et al. Which chronic obstructive pulmonary disease care recommendations have low implementation and why? A pilot study. BMC Res Notes 2012;5:652.

15. Barr RG, Celli BR, Martinez FJ, et al. Physician and patient perceptions in COPD: the COPD Resource Network Needs Assessment Survey. Am J Med 2005;118:1415.e9-e17.

16. Yawn BP, Wollan PC. Knowledge and attitudes of family physicians coming to COPD continuing medical education. Int J Chron Obstruct Pulmon Dis 2008;3:311-7.

17. Johnston CL, Maxwell LJ, Maguire GP, et al. How prepared are rural and remote health care practitioners to provide evidence-based management for people with chronic lung disease? Aust J Rural Health 2012;20:200-7.

18. Perez X, Wisnivesky JP, Lurslurchachai L, et al. Barriers to adherence to COPD guidelines among primary care providers. Respir Med 2012;106:374-81.

19. Keating A, Lee A, Holland AE. What prevents people with chronic obstructive pulmonary disease from attending pulmonary rehabilitation? A systematic review. Chron Respir Dis 2011;8:89-99.

20. Thorpe O, Johnston K, Kumar S. Barriers and enablers to physical activity participation in patients with COPD: a systematic review. $J$ Cardiopulm Rehabil Prev 2012;32:359-69.

21. Mathar H, Fastholm P, Hansen IR, et al. Why Do Patients with COPD Decline Rehabilitation. Scand J Caring Sci 2016;30:432-41.

22. Cox NS, Oliveira CC, Lahham A, et al. Pulmonary rehabilitation referral and participation are commonly influenced by environment, knowledge, and beliefs about consequences: a systematic review using the Theoretical Domains Framework. J Physiother 2017;63:84-93.

23. British Standards Institute. BS 7000-6:2005: Design management systems - Managing Inclusive Design - Guide. London: British Standards Institute, 2005.

24. John Clarkson P, Coleman R. History of Inclusive Design in the UK. Appl Ergon 2015;46:235-47.

25. Clarkson PJ, Keates S. User capabilities and product demands. In Proceedings of the International Conference on Inclusive Design and Communications, 2003:384-9. 
26. Maddocks M, Kon SS, Canavan JL, et al. Physical frailty and pulmonary rehabilitation in COPD: a prospective cohort study. Thorax 2016;71:988-95.

27. Pierobon A, Sini Bottelli E, Ranzini L, et al. COPD patients' selfreported adherence, psychosocial factors and mild cognitive impairment in pulmonary rehabilitation. Int $J$ Chron Obstruct Pulmon Dis 2017;12:2059-67.

28. Panella M, Marchisio S, Di Stanislao F. Reducing clinical variations with clinical pathways: do pathways work? Int J Qual Health Care 2003;15:509-21.

29. Persad U, Langdon P, Clarkson J. Characterising user capabilities to support inclusive design evaluation. Universal Access in the Information Society 2007;6:119-35.

30. Stanton NA, Salmon PM, Walker GH, et al. Human factors methods: a practical guide for engineering and design. Aldershot, UK: Ashgate, 2005.

31. Grundy M, Ahlburg D, Ali M, et al. Research report 94: Disability in Great Britain. London: Corporate Document Services, 1999.

32. Waller SD, Langdon PM, Clarkson PJ. Using disability data to estimate design exclusion. Universal Access in the Information Society 2010;9:195-207.

33. Waller SD, Bradley MD, Langdon PM, et al. Visualising the number of people who cannot perform tasks related to product interactions. Universal Access in the Information Society 2013;12:263-78.
34. Engineering Design Centre. Exclusion Calculator. http://calc.inclusive designtoolkit.com/

35. Smith J, Firth J. Qualitative data analysis: the framework approach. Nurse Res 2011:18:52-62.

36. Warren CAB. 4 Qualitative Interviewing. Handbook of Interview Research 2002:83-102.

37. Mason M. Sample Size and Saturation in PhD Studies Using Qualitative Interviews. Forum: Qualitative Social Research 2010;11 http://www.qualitative-research.net/index.php/fqs/article/view/1428/ 3027

38. Baker SE, Edwards R. How many qualitative interviews is enough? National Centre for Research Methods Review Paper. 2012 http:// eprints.ncrm.ac.uk/2273/ (accessed 20 May 2016).

39. Craig P, Dieppe P, Macintyre S, et al. Developing and evaluating complex interventions: the new Medical Research Council guidance. BMJ 2008;337:a1655.

40. Waller S, Bradley M, Hosking I, et al. Making the case for inclusive design. Appl Ergon 2015;46:297-303.

41. Liu Y, Dickerson T, Wallker SD et al. The Role of Inclusive Design in Improving People's Access to Treatment for Back Pain. In: Langdon P, Lazar J, Heylighen A, Dong H. eds. Breaking Down Barriers. CWUAAT 2018. Switzerland: Springer, Cham, 2018.

42. Johnson D, Clarkson J, Huppert F. Capability measurement for Inclusive Design. Journal of Engineering Design 2010;21:275-88. 